\title{
Satellite altimeters for detection of oceanic internal waves
}

José C. B. da Silva

A novel synergetic approach enables the identification of largeamplitude, short-period internal solitary waves from high-rate satellite altimeter data.

Internal waves (IWs) are waves that exist within the body of a density-stratified fluid. During summer, the top $20-30 \mathrm{~m}$ of the ocean can be up to $10^{\circ} \mathrm{C}$ warmer than the water below and this gives rise to a thermocline along which IWs can travel. The same is true-year-round-in the tropical ocean, where the mixed top layer $(100-200 \mathrm{~m})$ is more buoyant than the deeper, denser water. IWs are therefore important for understanding the evolution of the climate system, i.e., they are thought to play an important role in determining the near-surface sea temperature structure and air-sea exchange processes. Furthermore, IWs are highly nonlinear features that can exceed $100 \mathrm{~m}$ in height (vertical peakto-trough distance) and that resemble solitary waves (solitons) with a permanent form (because of a balance between nonlinear cohesive and linear dispersive forces in the water). ${ }^{1}$ They are thus also known as internal solitary waves (ISWs), i.e., often appearing as trains or packets of waves whose individual periods can exceed 30 minutes (and whose wavelengths are typically 3$10 \mathrm{~km}$, in tropical seas). Although ISWs travel in the interior of the ocean as disturbances of density, they are not associated (to the first order) with an elevation or depression of the sea surface (i.e., they cause a maximum elevation/depression of the surface of only a few tens of centimeters). It is therefore difficult to detect ISWs with standard satellite altimeters.

The first space-based observations of ISWs were made from color photographs (obtained during the 1975 Apollo-Soyuz mission), which showed $100 \mathrm{~km}$-long stripes on the ocean surface. ${ }^{2}$ Satellite radar imaging of the sea surface, however, allows much better identification of ISWs. In such data sets, the ISWs appear as bright and dark bands on the gray radar clutter. This is a result of the enhanced and reduced roughness produced by convergent and divergent surface currents that interact with the surface waves (see Figure 1 ). ${ }^{3}$ Conventional satellite radar altimeters are known for their extraordinary ability to measure

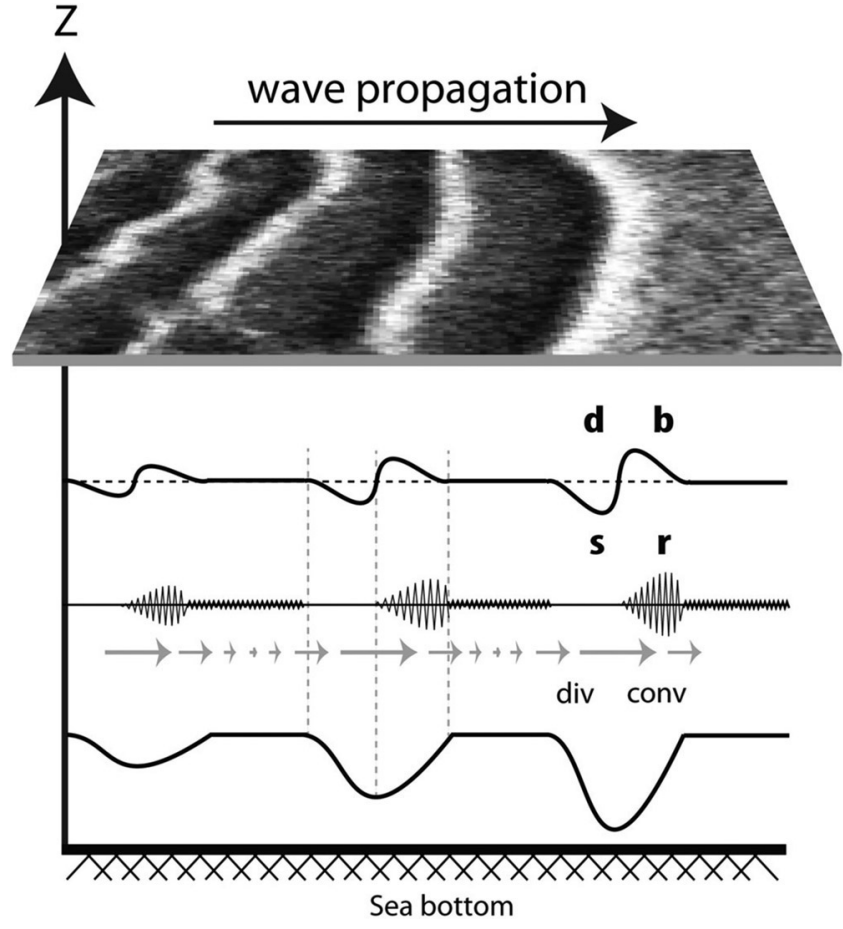

Figure 1. The propagation-from left to right-of internal waves (IWs). The top image is an example of grayscale satellite radar data. From top to bottom, the top profile is a synthetic aperture radar (SAR) intensity profile along the IWs, where bright, enhanced backscatter (b) precedes dark, reduced backscatter (d) in the direction of propagation. The next profile is a surface roughness representation that indicates how rough ( $r$ ) and smooth (s) the surface is along an IW train. The third profile depicts the surface current variability that is induced by the IWs, i.e., an indication of convergence (conv) and divergence (div) fields near the surface. The bottom profile shows the density perturbation produced by the IW propagation.

sea surface height anomalies (on the order of a few centimeters). These nadir-pointing instruments, however, provide along-track measurements with ground spatial resolution of only about $10 \mathrm{~km}$. 
A new generation of altimeters-such as those on the Jason-2/Jason-3 satellites-operate at higher measurement rates $(20 \mathrm{~Hz})$ than the conventional instruments. The new altimeters therefore offer better effective spatial resolution (about $300 \mathrm{~m}$ ). In our work, ${ }^{4}$ we have shown that it is theoretically possible to detect ISWs from a number of short-period signatures within the new high-resolution data sets. There are two factors that need to be considered for such detection of a measurable signal from the surface roughness manifestations of short-period IWs. First, it is theoretically predicted that differential scattering from the dual-band Jason-2/3 altimeter should be useful for isolating the contribution of small-scale surface waves to mean-square slope variations that are caused by ISWs. ${ }^{5}$ Current re-tracking algorithms (based on the Brown model ${ }^{6}$ ), however, may generate unphysical artifacts in response to inhomogeneities in backscatter strength. ${ }^{7}$

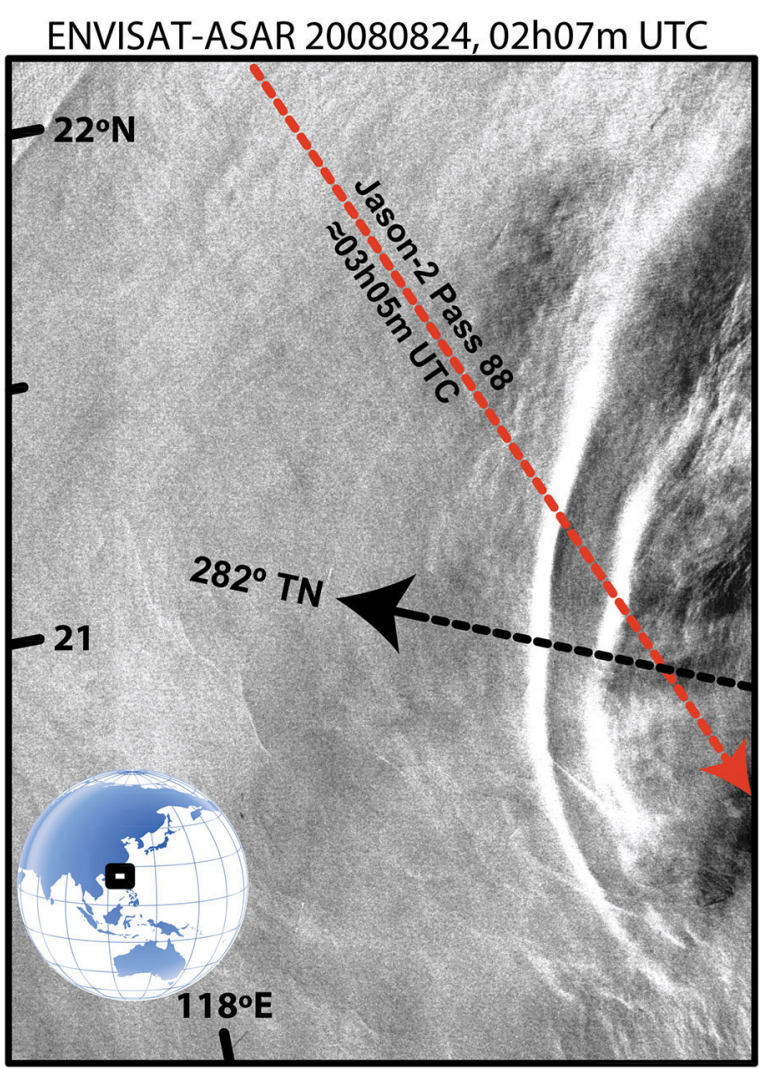

Figure 2. Image from the Advanced Synthetic Aperture Radar instrument on Envisat, acquired on 24 August 2008 at 02:07 UTC, showing strong surface manifestations of IWs. The red line indicates the ground track of the Jason-2 satellite, which carries an altimeter. The black arrow denotes the propagation direction of the internal solitary wave train, i.e., $282^{\circ}$ from true north (TN).
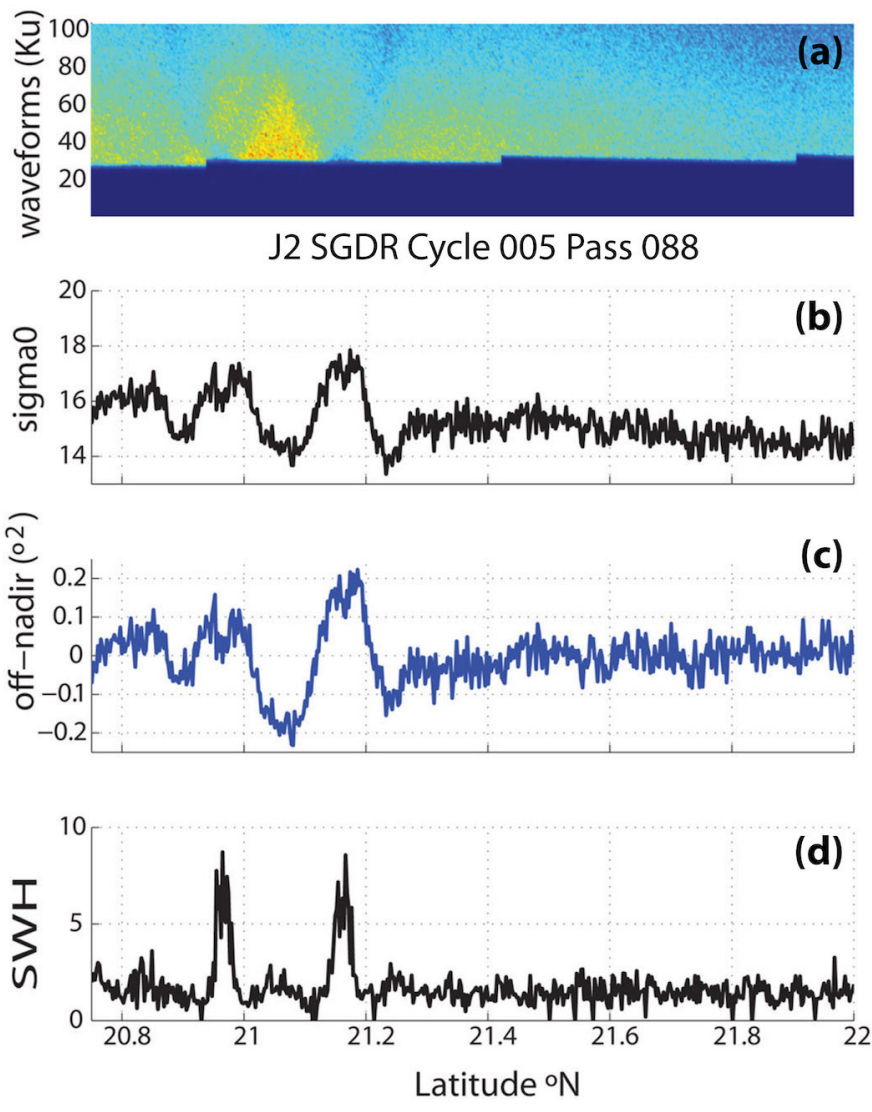

Figure 3. (a) Radargram showing the along-track IW signatures in high-rate $(20 \mathrm{~Hz})$ altimeter data from Jason-2 (J2) sensor geophysical data records (SGDRs). (b) Along-track normalized backscatter (sigma0) radar altimeter perturbations in the Ku-band. (c) Modulation of the instrument's off-nadir angle. (d) Significant wave height (SWH) profile.

Our observation method for the unambiguous recognition of ISWs is a synergetic approach in which we deal with synthetic aperture radar (SAR) and other high-resolution imaging sensors. ${ }^{4}$ We obtain the mean-square slope directly from normalized backscatter (sigma0) along-track records. In addition, we use differential scattering from the dual-band $(\mathrm{Ku}-$ and $\mathrm{C}$ bands) microwave pulses of the Jason-2 high-rate radar altimeter to identify ISWs in the along-track data records. We also propose a new method for the identification of ISWs in high-rate radar altimeter records. For this, we combine along-track differenced mean-square slopes across ISW crests and we edit the waveform shape variation. 
To validate our methodology, we have examined quasicoincident (in time and space) SAR images of ISWs in various deep-ocean regions. For example, at 02:44 UTC on 24 August 2008, Jason-2 overpassed the South China Sea (pass 088, cycle 005) to the west of the Luzon Strait, where large-amplitude ISWs are known to be generated. ${ }^{8}$ An image from the Envisat Advanced SAR instrument (acquired at 02:07 UTC) for the same location-see Figure 2-reveals a large ISW train propagating to the west, with wavefronts that extend for at least $200 \mathrm{~km}$ across the Jason-2 data ground track (red line in Figure 2).

We also show the geophysical parameters obtained from the altimeter's sensor geophysical data records (SGDRs) in Figure 3 (for the same time period/location). Of particular interest-see Figure 3(b) - are the along-track radar altimeter backscatter perturbations (in the Ku-band) between 20.8 and $21.4^{\circ} \mathrm{N}$ that coincide with a SAR signature of ISWs. These signatures also coincide with parabolic-like signatures in the radargram and with a significant modulation in the off-nadir angle, as seen in Figure 3(a) and (c), respectively. We also note two peaks in the significant wave height (SWH): see Figure 3(d). This kind of synergetic analysis provides strong observational evidence of ISWs in the different altimeter geophysical parameters, i.e., the apparent 'off-nadir angle,' sigma0 (retrieved from the maximum likelihood estimator algorithm version 4, MLE4), and SWH (also retrieved from MLE4). In addition, our analysis of the altimeter radargrams reveals parabolic-like features (sometimes called $\mathrm{v}$-shaped features) that are associated with short-scale sigma0 events and that can be modeled as sigma0 Dirac anomalies. ${ }^{7}$

In summary, we have developed a new, synergetic observation approach for the identification of internal solitary waves in the ocean from high-rate satellite altimeter data. Our results illustrate that oceanographers interested in short-period internal wave signals may find useful information in the $20 \mathrm{~Hz}$-rate Jason-2/3 altimeter products currently being generated, or in forthcoming data sets from Sentinel-3 and AltiKa. In our future research we plan to look at Sentinel-3 Ku/C Radar Altimeter (SRAL) data, which will be acquired in exact synergy with ocean color data from the Ocean and Land Colour Instrument (OLCI). The OLCI and SRAL radar altimeter data will thus be acquired simultaneously over the same ground tracks andduring cloud-free conditions-provide an exact match between the altimeter and optical images. We also plan to use Jason-3 altimeter data to develop a new method for automatic detection of short-period internal waves in radar altimeter data.
Author Information

José C. B. da Silva

Department of Geoscience, Environment and Spatial Planning

University of Porto

Porto, Portugal

José da Silva is an associate professor. He holds a $\mathrm{PhD}$ in oceanography from the University of Southampton, United Kingdom. His research interests involve many aspects of satellite oceanography, in particular small-scale processes such as internal waves.

\section{References}

1. A. R. Osborne and T. L. Burch, Internal solitons in the Andaman Sea, Science 208, pp. 451-460, 1980.

2. J. R. Apel, J. R. Holbrook, A. K. Liu, and J. J. Tsai, The Sulu Sea internal soliton experiment, J. Phys. Oceanog., pp. 1625-1651, 1985.

3. W. Alpers, Theory of radar imaging of internal waves, Nature 314, pp. 245-247, 1985. 4. J. C. B. da Silva and A. L. F. Cerqueira, A note on radar altimeter signatures of internal solitary waves in the ocean, Proc. SPIE 9999, p. 99902, 2016. doi: $10.1117 / 12.2240870$.

5. B. Chapron, K. Katsaros, T. Elfouhaily, and D. Vandemark, A note on relationships between sea surface roughness and altimeter backscatter, in B. Jahne and E. C. Monahan (eds.), Air-Water Gas Transfer, pp. 869-878, AEON Verlag \& Studio, 1995.

6. G. S. Brown, The average impulse response of a rough surface and its application, IEEE Trans. Antennas Propag. 25, pp. 67-74, 1977.

7. G. Dibarboure, F. Boy, J. D. Desjonqueres, S. Labroue, Y. Lasne, N. Picot, J. C. Poisson, and P. Thibaut, Investigating short-wavelength correlated errors on lowresolution mode altimetry, J. Atmos. Oceanic Technol. 31, pp. 1337-1362, 2014.

8. M. H. Alford, T. Peacock, J. A. MacKinnon, J. D. Nash, M. C. Buijsman, L. R. Centuroni, S.-Y. Chao, et al., The formation and fate of internal waves in the South China Sea, Nature 521, pp. 65-71, 2015. 